\title{
Mesures de contraintes en écoulement cavitant dans les pales de l'inducteur oxygène du moteur fusée Vulcain
}

\author{
J. Kermarec CETIM Nantes
}

J. Desclaux et F. Martignac SEP Vernon

\section{Introduction}

Les turbopompes hydrogène et oxygène liquides du moteur fusée Vulcain comportent chacune un inducteur. Ceux-ci sont caractérisés par des performances en cavitation et des vitesses périphériques élevées. De plus, aux conditions nominales de fonctionnement de la turbopompe oxygène, la charge sur les pales de l'inducteur est élevée.

C'est pourquoi dans le cadre du programme préparatoire de développement du moteur mené sous maîtrise d'œuvre du Centre national d'études spatiales (CNES) pour le compte de l'Agence spatiale européenne (ESA), la Société européenne de propulsion (SEP) a réalisé un inducteur oxygène baptisé inducteur technologique ; elle a élaboré pour cet inducteur un plan d'essais à l'eau en vue de sa caractérisation hydrodynamique en conditions de similitude, et de l'évaluation des contraintes mécaniques réelles dans les pales.

Néanmoins le fluide d'essai, l'eau froide, présente l'inconvénient de ne pas avoir d'effet thermodynamique en cavitation, ce retard à la cavitation étant constaté avec les ergols.

Les essais ont été réalisés en 1986 à l'Etablissement de Nantes du CETIM grâce à une collaboration étroite entre les équipes SEP et CETIM.

\section{Moyens d'essais}

L'objectif de l'étude étant essentiellement de connaitre le comportement de l'inducteur en cavitation, la boucle d'essais, les moyens de mesures et les critères d'essais ont été choisis en conséquence.

\subsection{Boucle d'essais}

La boucle d'essais est en circuit fermé (fig. 1) avec les caractéristiques suivantes :

- réglage possible du $N P S H$ disponible de 1 à $45 m C E$; - désaération de l'eau par fonctionnement prolongé sous vide ;

— possibilité de fonctionner longtemps en cavitation avancée, ce qui nécessite un temps de transit suffisant pour que la cavitation soit résorbée en un cycle ;

- entraînement par moteur à vitesse variable et multiplicateur à $8000 \mathrm{tr} / \mathrm{min}$ avec un faible niveau de vibration.

En plus des grandeurs nécessaires à la détermination des caractéristiques de l'inducteur (débit, couple, hauteur d'élévation globale, vitesse de rotation), la mesure de la pression moyenne à l'aspiration dans une section à deux fois le diamètre de l'inducteur est prévue sur l'installation pour la détermination du NPSH.

Cavitating flow constraint measurements in the oxygen inductor blades of a Vulcain rocket engine.

Readjustment of the calculation of hydraulic loads on the inductor blades is obtained from a test programme carried out at CETIM in Nantes on a rocket engine pump technological inductor on which 13 strain gauges are fitted.

In addition to the conventional measurements (height characteristics, torque, yield and NSPH depending on the flow rate), the signals of gauges and transient pressure gauge heads are recorded during NPSH drops until a hoisting height significant fall is obtained. The data processing comprises frequency analysis spectrum, transient pressure and constraint evolutions depending on the NPSH, and a correlation with the cavitation figures photographed. 


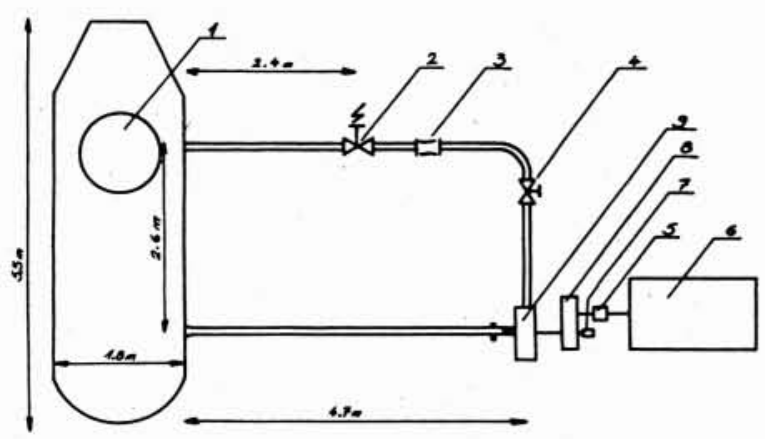

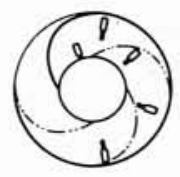

Pale $n^{*} 1$

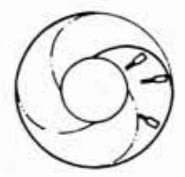

Pale $n * 2$

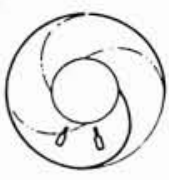

Pale $n^{\circ} 3$

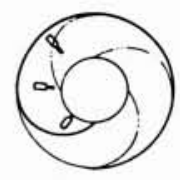

Pale $n^{\circ} 6$

5.

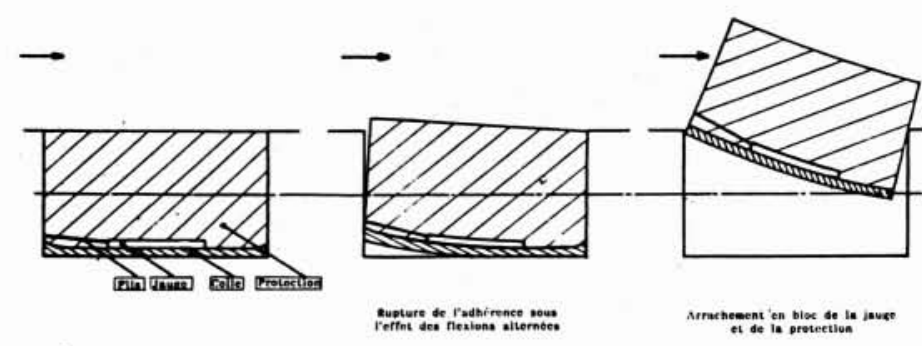

6.

1. Schéma de la boucle hydraulique.

1. Ballon pressurisation. 2. Vanne de réglage. 3. Débitmètre.

4. Vanne de décharge. 5. Couplemètre. 6. Moteur $350 \mathrm{~kW} .7$.

Collecteur tournant. 8. Multiplicateur. 9. Modèle.

2. Schéma du montage d'essais d'inducteur.

1. Manchette. 2. Bulbe. 3. Arbre creux. 4. Inducteur. 5.

Redresseur. 6. Refoulement. 7. Volute. 8. Accouplement.

3. Implantation des mesures.

4. Schéma de l'empreinte usinée.

5. Implantation des jauges de contrainte.

6. Processus d'arrachement des jauges de contrainte.

7. Configuration finale de l'implantation des jauges.

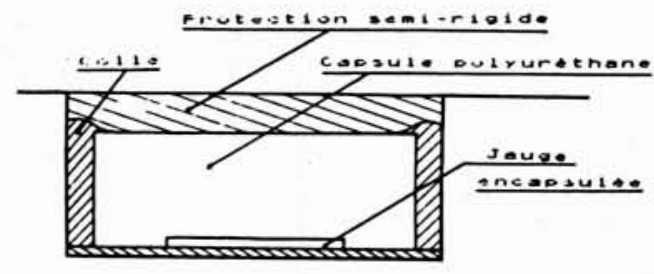

7. 


\subsection{Montage d'essais}

L'inducteur à oxygène liquide est une pompe axiale à 4 pales de diamètre extérieur $155 \mathrm{~mm}$ et d'angle moyen au bord d'attaque de $13.5^{\circ}$. Il est installé dans un montage (fig. 2) spécialement conçu pour ces essais. L'arbre creux permet le passage des fils de jauges implantées sur les pales. Une manchette cylindrique réalisée en altuglass permet, au moyen d'un éclairage stroboscopé, la visualisation des figures de cavitation à travers un prisme collé.

$\mathrm{La}$ vitesse de rotation maximale de l'ensemble est de $8000 \mathrm{tr} / \mathrm{min}$ pour une pression de sortie de l'ordre de 10 bar.

Pour suivre l'évolution de l'écoulement dans la machine, ce montage comporte des prises pariétales de pression statique en valeurs moyennes (14) et instantanées ( 5 capteurs piézoélectriques) (fig. 3 ). Trois accéléromètres au droit des paliers permettent de suivre les vibrations dans les trois axes.

Afin de relever les contraintes dans les pales de l'inducteur et pour respecter leur profil, la mise en place des jauges a nécessité l'usinage par électroérosion d'emplacements limités par l'épaisseur des pales (fig. 4).

La rainure permet le cheminement des fils jusqu'à l'arbre creux, puis au collecteur tournant qui transmet six signaux simultanément. La jauge collée au fond de la cavité est recouverte d'un produit servant de protection et d'étanchéité. Leur implantation dans l'inducteur est donnée par la figure 5 .

\subsection{Tenue des jauges de contraintes}

Il est apparu en cours d'essais des problèmes liés à la tenue des jauges. Des mesures embarquées de ce type avaient déjà été faites par le CETIM mais pas dans des conditions aussi sévères de vitesse et cavitation. La principale cause de détérioration des jauges en fonctionnement s'est avérée être l'effet d'arrachement créé par des vitesses importantes d'écoulement sur une paroi déformable soumise à des sollicitations périodiques.

Des essais préliminaires avaient été réalisés, avant l'étude elle-même, afin de tester les qualités de différentes colles et de différents revêtements protecteurs dans des écoulements de vitesse équivalente aux essais d'inducteur (environ $45 \mathrm{~m} / \mathrm{s}$ ). Les essais ont été effectués sur des éprouvettes statiques car le facteur principal d'agression était supposé être a priori la cavitation. Ces tests avaient permis la sélection d'un revêtement dur résistant à l'érosion cavitante pendant plus de 12 heures.

Les premiers essais en rotation se révélèrent catastrophiques, la tenue des jauges n'excédant pas quelques minutes. Le processus de détérioration qui a pu être observé par la suite semble être le suivant (fig. 6) :

Une analyse de ce processus et une nouvelle recherche de produits avec essais de simulation sur des éprouvettes en flexions alternées puis tests de plusieurs solutions sur l'inducteur aboutirent au compromis suivant (fig. 7) :

La jauge utilisée est préconditionnée dans une capsule souple de polyuréthane découpée à la taille de l'empreinte puis collée. La couche de protection fine est assurée par un produit de bonne adhérence, présentant un compromis dureté-flexibilité convenable.
Cette solution donnait une durée de vie de l'instrumentation de une à deux heures à pleine vitesse. Ces temps permettaient d'effectuer plusieurs points de mesures mais nécessitaient plusieurs instrumentations successives de l'inducteur pour mener à bien l'étude complète.

\subsection{Transmission des informations}

L'instrumentation du rotor nécessite la transmission des signaux par la mise en œuvre d'un procédé (télémesure ou collecteur tournant). C'est le deuxième qui a été retenu après examen des points suivants :

- nombre important des informations à transmettre dans une bande de fréquence dépassant $2 \mathrm{kHz}$;

- peu de volume disponible dans le rotor;

- vitesse de rotation élevée nécessitant un très bon équilibrage dynamique du rotor ;

- l'utilisation d'un multiplicateur pour l'entraînement de l'inducteur permettant de monter en bout d'arbre grande vitesse un collecteur de petit diamètre.

Cette solution s'est avérée satisfaisante à l'usage malgré quelques problèmes d'échauffement à la mise au point, permettant la réalisation de mesures dynamiques correctes dans un environnement industriel (parasites).

\subsection{Procédure d'essais}

Pour étudier les phénomènes liés à la cavitation, la première procédure utilisée était de baisser le NPSH disponible par paliers et ensuite de procéder à l'acquisition des mesures une fois le régime stabilisé. Cette méthode n'a pu être mise en œuvre très longtemps car elle conduisait à un temps de fonctionnement trop long pour la durée de vie de l'instrumentation en jauges.

Pour raccourcir le temps d'essais, la procédure adoptée a été de procéder à l'enregistrement direct de toutes les informations, y compris la pression absolue à l'aspiration qui sert de repère pour définir le $N P S H$ disponible, alors que la pression de la boucle diminue de manière continue jusqu'à atteindre les conditions de pleine cavitation. La durée d'une séquence est de l'ordre de $5 \mathrm{~min}$ pour une variation de la pression de $20 \mathrm{mCE}$ environ.

\section{Résultats}

\subsection{Coefficients adimensionnels utilisés}

Débit :

$$
\varphi=\frac{c}{u}=\frac{\text { vitesse débitante }}{\text { vitesse périphérique }}
$$

Hauteur :

$$
\psi=\frac{H M T}{\rho \cdot \omega^{2} \cdot R^{2}}
$$

Couple :

$$
C=\frac{C}{\rho \cdot \omega^{2} \cdot R^{5}}
$$




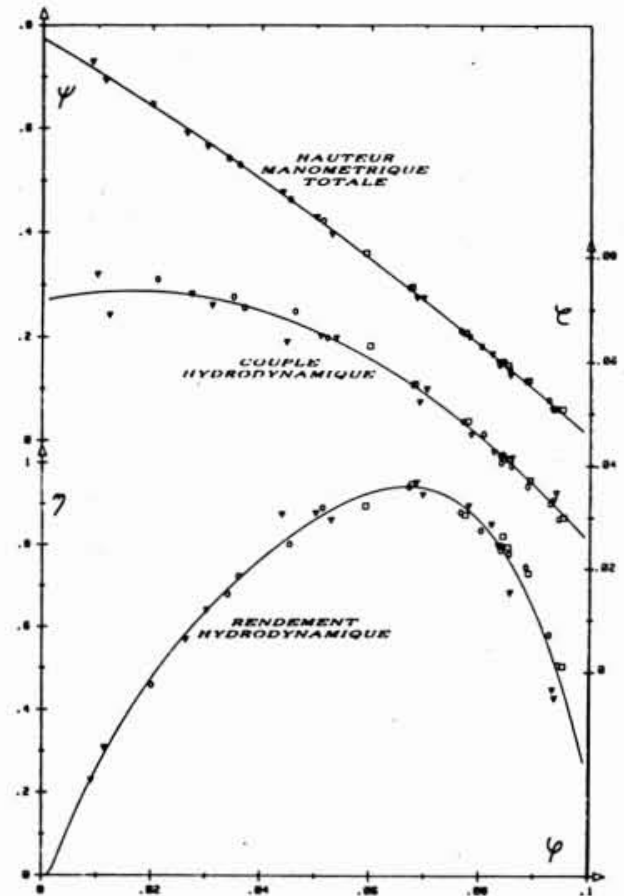

8. Caractéristiques fonctionnelles en hauteur manométrique totale, couple et rendement hydrodynamique.

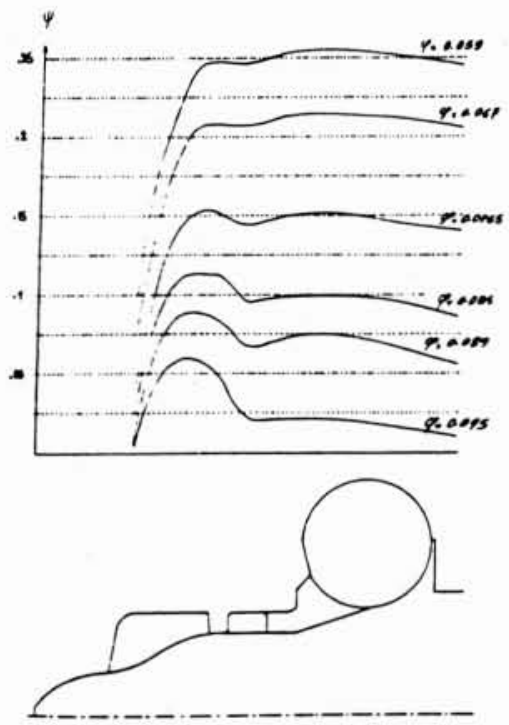

9. Evolution de la pression statique moyenne pariétale entre l'aspiration et le refoulement.

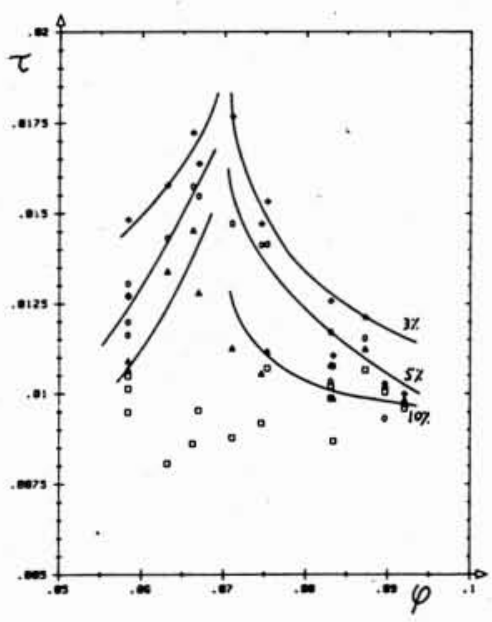

10. Caractéristique en cavitation, NPSH à différents taux de chute de performance en fonction du débit.
NPSH :

$$
\tau=\frac{N P S H}{\rho \cdot \omega^{2} \cdot R^{2}}
$$

Contraintes :

$$
\sigma^{*}=\frac{\sigma}{\rho \cdot \omega^{2} \cdot R^{2}}
$$

\subsection{Caractérisation hydrodynamique}

Ces essais ont consisté en l'établissement des performances hydrodynamiques de l'inducteur de vitesse spécifique $N_{s}=1.86$ (S.I.) sur une plage de débit de 12 à $112 \%$ du débit nominal. Ils ont abouti au tracé de la figure 8 .

Ces caractéristiques sont typiques des inducteurs (forte pente pour la $H M T$ ) ; le rendement présente de fortes valeurs dont le maximum (p.m.r.) est à $80 \%$ du débit nominal, les inducteurs étant dimensionnés pour la performance en cavitation. Les essais à 3 vitesses de rotation montrent une bonne indépendance vis-à-vis de cette dernière.

En plus des mesures globales, 15 prises de pression pariétale permettent de suivre l'évolution de la pression statique entre l'aspiration et le refoulement (fig. 9).

Cette figure montre le comportement général d'un inducteur (forte charge à l'entrée suivie d'un palier et d'une chute de pression en sur-débit). En aval de l'inducteur, les éléments suivants (redresseur et volute) sont en servitude et ne présentent donc aucun intérêt du point de vue hydraulique.

\subsection{Cavitation}

Les mesures de hauteur d'élévation statique de l'inducteur à débit et vitesse constants en fonction de la pression à l'aspiration jusqu'à un régime de cavitation développée, ont permis de tracer la figure 10 à $8000 \mathrm{tr} / \mathrm{min}$ où sont représentées les variations du NPSH en fonction du débit pour des chutes de hauteurs d'élévation statique de $3,5,10,20 \%$ de la valeur en régime non cavitant.

Ces courbes présentent des valeurs minimales autour du débit nominal avec un pic vers $80 \%$ de ce dernier. Les instabilités de fonctionnement en forte cavitation conduisent à des résultats pour $20 \%$ très dispersés. Les limites du banc n'ont pas permis d'atteindre la remontée des courbes en forts débits.

Les figures de cavitation associées à ces performances peuvent être scindées en deux types : cavitation d'entrefer et cavitation de bord d'attaque. Le premier type est un tourbillon de bulles généré par l'écoulement passant de l'intrados à l'extrados à la périphérie de l'aube, pratiquement toujours présent à $8000 \mathrm{tr} / \mathrm{min}$ quel que soit le NPSH. Il a tendance à remonter vers l'aspiration surtout en sous-débit avec la recirculation du noyau externe de l'écoulement. Avec la diminution de la pression d'aspiration, les tourbillons s'étendent vers l'aval jusqu'à atteindre le bord de fuite. Le deuxième type est caractérisé par des filets de bulles attachés au bord d'attaque côté extrados, et qui ne semblent pas avoir de point de rattachement sur la pale. Sur cet inducteur, la zone la plus extérieure du bord d'attaque 
cavite en premier, cette poche de cavitation s'étend vers le moyeu avec la diminution de la pression d'aspiration.

Les performances de l'inducteur vis-à-vis d'une valeur de NPSH peuvent être reliées à un état de cavitation grâce à la visualisation. On distingue plusieurs phénomènes localisés sur la figure 11.

Dans la zone 1, seule la cavitation d'entrefer est présente et s'allonge avec la diminution de la pression d'aspiration. La zone 2 voit l'apparition des premiers filets de bulles accrochés au bord d'attaque qui recouvrent la partie visible de la pale avec la décroissance du NPSH jusqu'à atteindre la zone de recouvrement de deux pales appelée le col. La cavitation d'entrefer dans la zone 3 puis la poche dans la zone 4 fluctuent autour du col et engendrent de fortes vibrations. Quand les deux types de cavitation ont passé le col (zone 5), l'écoulement se stabilise et l'inducteur est entièrement recouvert par la cavitation.

\subsection{Contraintes dans les pales}

Cette publication intervenant en cours de dépouillement des valeurs instantanées, nous ne présentons ici que quelques résultats concernant les contraintes dans les pales.

Les micro-déformations mesurées par les jauges extensométriques ne permettent pas de connaître l'état de contrainte de la pale au niveau de l'empreinte, car la mesure n'a été réalisée que dans la direction radiale de l'inducteur. Elles mesurent les déformations en flexion dues aux efforts de surpression, ainsi que les déformations en traction engendrées par l'effort centrifuge.

Sur la figure 12, sont représentées en fonction de la pression amont pour 4000 et $6000 \mathrm{tr} / \mathrm{min}$, les fluctuations de contraintes dont les valeurs moyennes sont centrées. D'une façon générale, les amplitudes des fluctuations varient rapidement en régime cavitant.

La figure 13 représente pour deux pressions amont très différentes (dont une en régime cavitant), la valeur des contraintes mesurées en fonction de la distance à l'entrée de l'inducteur. Les fluctuations des contraintes augmentent lorsque la pression amont diminue pour une contrainte moyenne maximale située aux environs de la zone de recouvrement des pales.

L'analyse fréquentielle menée sur les signaux est qualitative appelant quelques remarques issues de l'observation des signaux temporels et de l'amplitude des spectres de Fourier, sur la bande $0-2500 \mathrm{~Hz}$.

De manière générale, pour toutes les contraintes dont la figure 14 constitue un exemple de dépouillement, le signal temporel présente une périodicité correspondant à un tour, valeur qui se retrouve sur le spectre avec la raie synchrone $(67 \mathrm{~Hz}$ à $4000 \mathrm{tr} / \mathrm{min}, 100 \mathrm{~Hz}$ à $6000 \mathrm{tr} / \mathrm{min}$ et $133 \mathrm{~Hz}$ à $8000 \mathrm{tr} / \mathrm{min}$ ) et la suite d'harmoniques d'amplitude peu différente.

Lorsque la pression à l'aspiration décroît, la périodicité reste marquée avec une amplitude de la raie synchrone augmentée mais des harmoniques plus réduites.

Quelques spectres présentent toutefois certaines particularités. Sur la figure 15, l'apparition d'une raie à $600 \mathrm{~Hz}$ à $8000 \mathrm{tr} / \mathrm{min}$ pour un $N P S H$ relativement élevé est expliquée par des essais de chocs sur l'arbre qui nous ont permis d'identifier cette raie par valeur légèrement

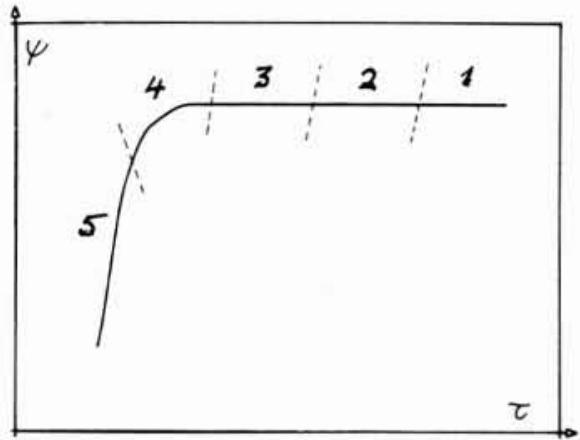

11. Observations reportées sur un exemple de courbe de cavitation (HMT vs NPSH).

supérieure comme le premier mode de flexion de l'arbre, dû au balourd de l'inducteur sur ses paliers (la différence est due à la diminution des fréquences par la masse d'eau ajoutée).

Pour un autre point de fonctionnement (correspondant à la zone 3 de la fig. 11), il apparaît une raie d'amplitude importante à la fréquence de $30 \mathrm{~Hz}$ sur plusieurs signaux (exemple sur la fig. 16), ce phénomène ayant été observé sur le site avec une fluctuation de la raie autour de cette valeur.

Cette vibration peut être expliquée par l'apparition de charges radiales dues au balourd hydraulique généré par les fluctuations des deux types de cavitation; ceci n'est qu'une hypothèse et demande à être confirmée par des investigations supplémentaires.

Afin de comparer les mesures avec le calcul, un modèle numérique a été réalisé à l'aide du code SAMCEF où une aube a été modélisée par éléments finis de coques planes. Les charges prises en compte sont les efforts centrifuges et les charges hydrauliques. Expérimentalement, on constate la répartition des contraintes moyennes données par le calcul comme le montre la figure 17.

Nous observons une contrainte faible à l'entrée car le gain de pression est faible et une contrainte maximale pour une pression importante, croissante dans une zone de la pale dont la résistance est affaiblie par l'affûtage. Puis une valeur relativement faible car la pression est décroissante en sortie d'inducteur pour une zone de pale très rigide. Ceci met bien en évidence la nécessité du compromis à trouver entre la finesse des aubages pour obtenir les meilleures performances et leur épaisseur minimale pour la tenue structurale en conditions cavitantes. 
616

J. KERMAREC et al.

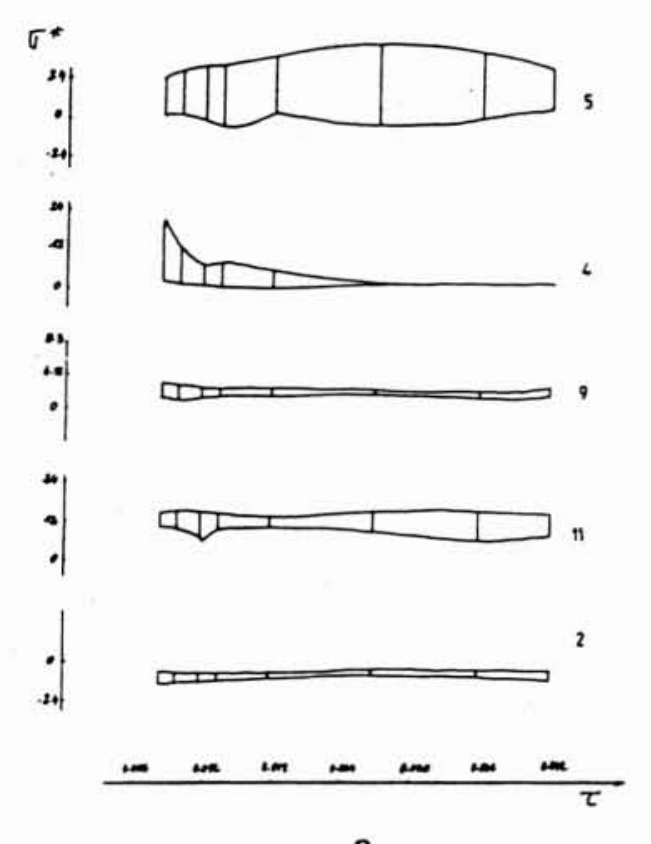

12.
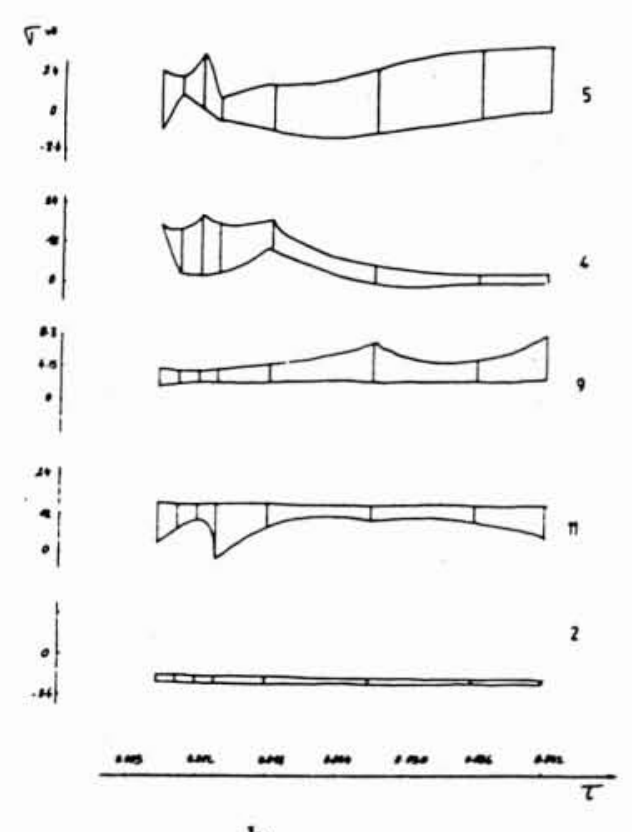

b
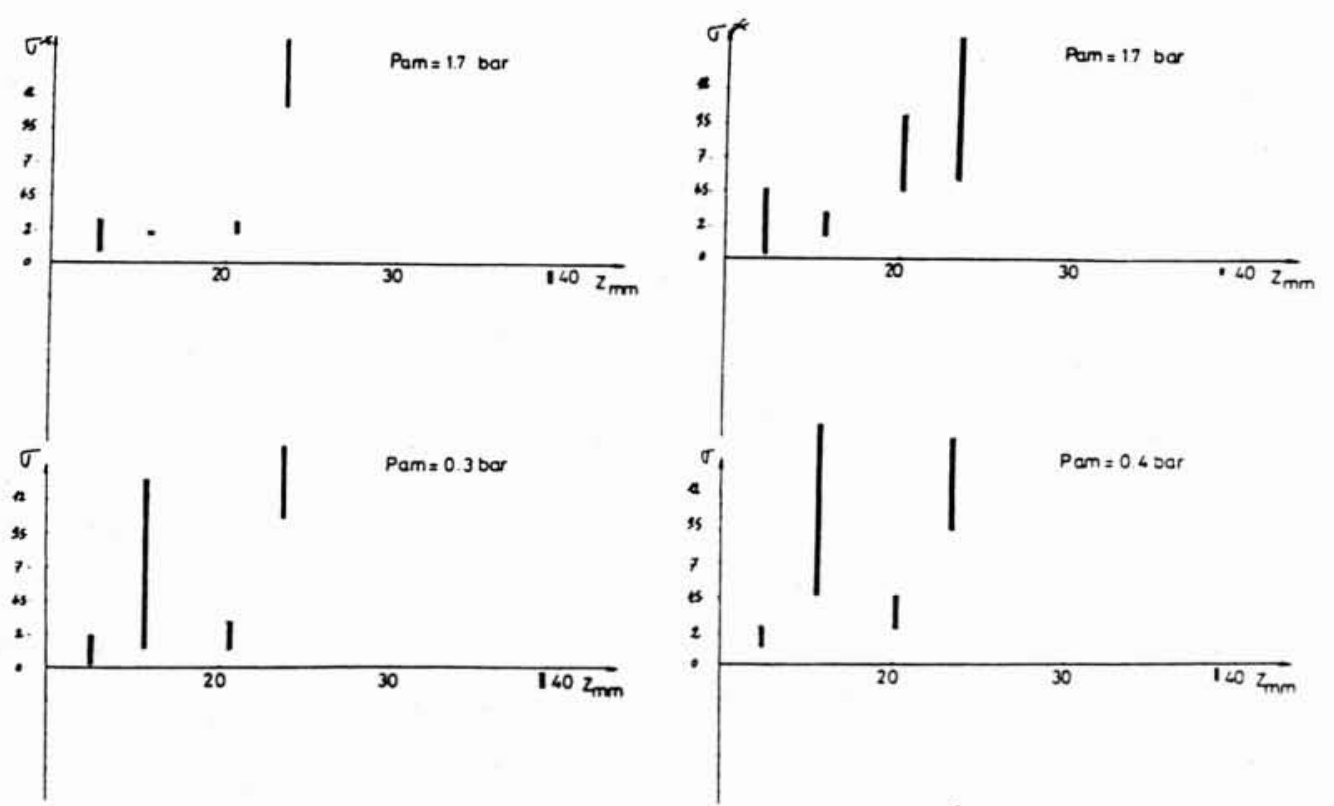

13.

a

b
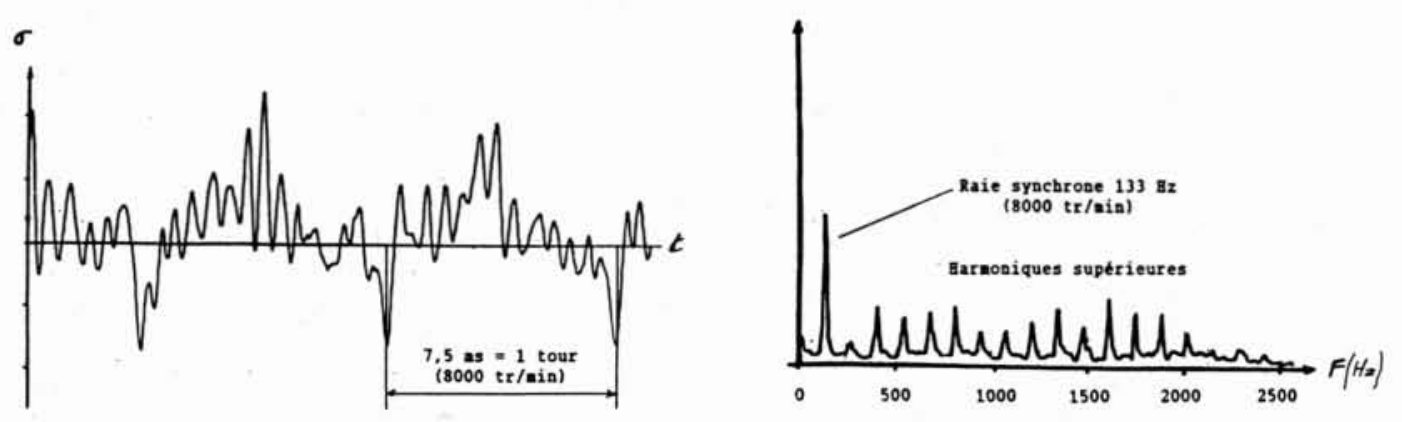

14.

a

b

LA HOUILLE BLANCHE/N 7/8-1988 
15.

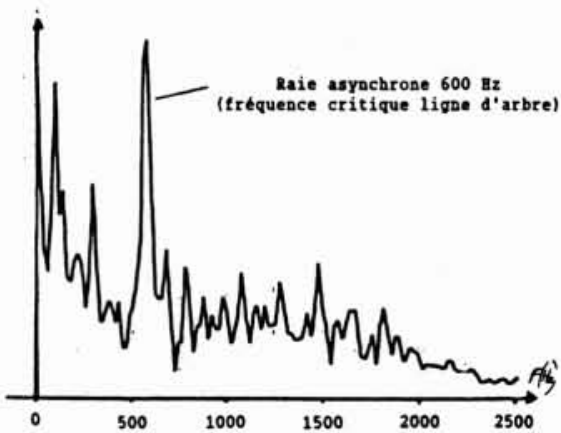

16.

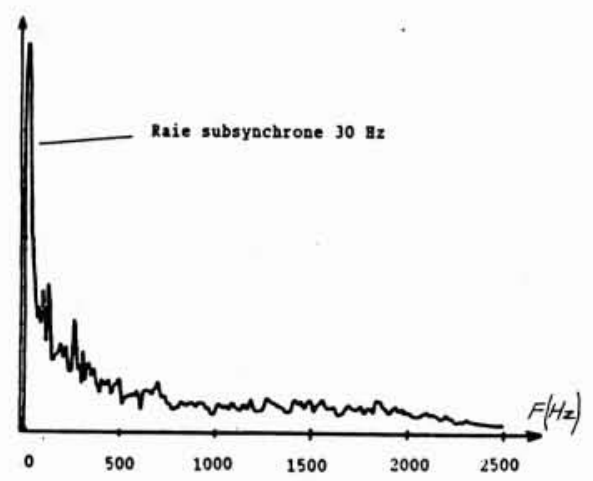

12. Valeurs moyennes et fluctuations des contraintes en fonction de la pression d'aspiration (a. 4000 tr/min, b. $6000 \mathrm{tr} / \mathrm{min})$.

13. Répartition des contraintes dans l'inducteur (a. 4000 tr/min, b. $6000 \mathrm{tr} / \mathrm{min})$.

14. Exemple de signal temporel (a) et de spectre (b) d'une mesure de contrainte dans l'inducteur.

15. Premier mode de flexion d'arbre retrouvé sur un signal de contrainte à $8000 \mathrm{tr} / \mathrm{min}$.

16. Apparition d'une raie à $30 \mathrm{~Hz}$ en écoulement cavitant fluctuant sur un signal de contrainte.

17. Comparaison théorie-expérience des contraintes moyennes dans les pales $(+e t \times$ : expérience -0 : calcul).

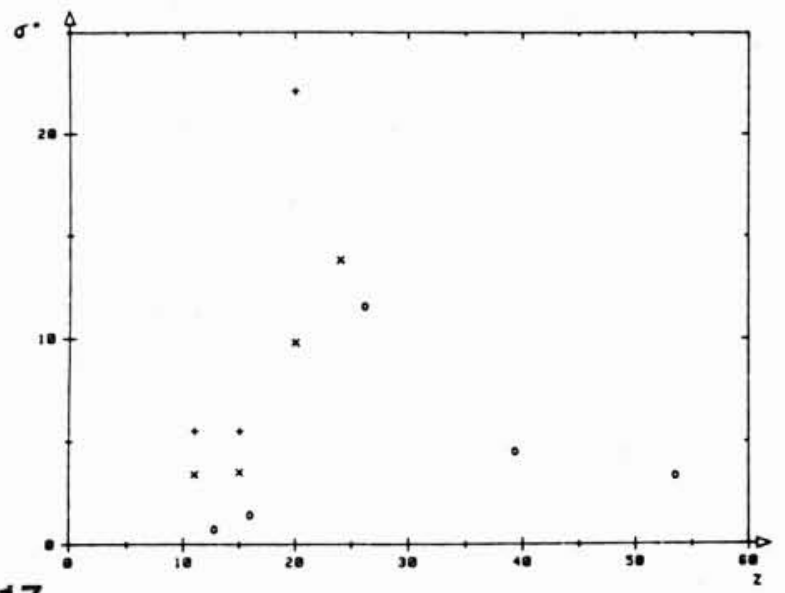

\section{Conclusion}

La campagne d'essais réalisée sur l'inducteur technologique en eau froide a amené bon nombre de résultats.

Les essais hydrauliques ont amené les confirmations suivantes :

- vérification de l'indépendance des caractéristiques fonctionnelles vis-à-vis de la vitesse de rotation, y compris en cavitation ;

- bonne concordance autour du point nominal entre la prévision théorique et les mesures ;

- connaissance du fonctionnement hors nominal de l'inducteur.

Les essais mécaniques ont fourni des signaux exploitables, où les raies harmoniques sont bien identifiées. Certains spectres ont mis en évidence des raies asynchrones dont une excitation du premier mode propre de flexion de la ligne d'arbre.

Les valeurs obtenues de contraintes seront confirmées par des traitements complémentaires ; par ailleurs après prise en compte des mesures de pressions pariétales instationnaires, des tentatives de corrélations des signaux de pression et de contrainte seront menées, ainsi qu'une étude plus précise des signaux accélérométriques.

La tenue des jauges de contraintes sur les pales a été le souci permanent de l'équipe réalisant les essais.
Adresses des auteurs :

Monsieur J. Kermarec

CETIM

BP 957

44076 Nantes Cedex 03

Tél. : 1640373635

Messieurs J. Desclaux

F. Martignac

SEP Vernon

BP 802

27207 Vernon

Tél. : 1632317200

17. 
M. LEFEBVRE : Envisagez-vous, par la recirculation du bord de fuite, de placer ce que les américains appellent un tranquillisateur, une sorte de chambre de recirculation qui va au-dehors du diamètre de tuyauterie ? Ceci permettrait d'avoir beaucoup moins de fluctuations et de cavitation dans les pales.

M. DESCLAUX: Pour répondre à votre première question, les dispositifs tranquillisateurs sur les conduites d'aspiration ont été testés par les américains. Il y a eu des publications à ce sujet et la NASA a également testé ces dispositifs sur certains inducteurs dans le domaine spatial. Il y a effectivement des avantages mais il y a aussi d'autres inconvénients. A l'heure actuelle, on n'envisage pas de les implanter mais c'est une solution de secours qui, a priori, n'est pas exclue si vraiment les problèmes sont critiques.

M. Colas: Avez-vous essayé de voir les fréquences modales d'ailettes? J'ai vu que vous aviez 630 ou $640 \mathrm{~Hz}$ sur le rotor mais, sur un corps complexe, il y a différentes fréquences modales et il serait intéressant de les avoir. Il serait bon notamment de voir si vous aviez un pic de fréquence qui correspondrait à cette fréquence modale d'ailettes, dans le spectre délivré par divers régimes hydrauliques.

J'ai vu aussi, d'après le film, que la traînée de cavitation était toujours dans le même sens. $\mathrm{Si}$ vous faites varier le débit, vous n'avez pas observé de passage de la cavitation de l'intrados à l'extrados, par exemple?

Dans ces problèmes, éventuellement sur des débits particuliers où l'on est, pour parler par métaphore, sur la lame de couteau, on peut avoir très rapidement le passage de la cavitation de l'intrados à l'extrados d'une manière alternative. On peut voir si, par hasard, cela pourrait répondre à une fréquence d'excitation des ailettes pour expliquer certaines destructions.

M. MARTIGNAC: En ce qui concerne les fréquences propres, on a effectué deux ou trois essais. L'inducteur était monté sur l'arbre et on a réalisé les mesures modales, mais je ne me rappelle plus les valeurs exactes. On avait obtenu deux pics à des fréquences relativement basses.

En ce qui concerne la cavitation intrados-extrados, on n'a pas eu de visualisation dans ce sens, mais je ne crois pas qu'on ait remarqué ce genre de chose. On a observé, en interprétant des mesures de pressions, qu'il y a de très fortes charges à l'entrée juste derrière le col côté extrados.
M. VERRY: Je voudrais faire une remarque à propos de votre réponse précédente. On a fait un certain nombre d'essais sur des ailettes qui sont peut-être moins poussées que les vôtres, avec des vitesses de rotation beaucoup plus classiques de 1500 à $2000 \mathrm{tr} / \mathrm{min}$. Mais il est certain que l'on a retrouvé un certain nombre de phénomènes que vous avez aussi mis en évidence.

En ce qui concerne ces programmes de fréquences propres, on avait instrumenté une hélice avec des jauges embarquées, le même type de manipulation que vous avez faite, avec des difficultés d'instrumentation moindres puisqu'il y avait des vitesses périphériques de l'ordre de $25 \mathrm{~m} / \mathrm{s}$. On avait mesuré les fréquences propres des pales en air et en eau. On a vu un déplacement de l'ordre de $15 \%$ des fréquences propres. Par contre, comme c'est le cas pour un cylindre immergé, on n'a pas modification de l'amortissement. En air ou en eau, c'est la même chose.

On a essayé de s'intéresser à une plage large de débit car, dans les applications qui nous intéressent à EDF, nous sommes parfois amenés à demander des fonctionnements à très faible débit, typiquement $10 \%$ du débit nominal.

On a donc essayé de regarder comment évoluaient les fluctuations de contraintes dans les pales avec des faibles débits. Comme vous l'avez mentionné, on arrive à des ordres de grandeur qui sont nettement supérieurs à ceux que l'on peut avoir au débit nominal (cinq à dix fois plus en valeur crête-à-crête).

D'autre part, on a essayé de regarder aussi comment transposer les fluctuations de contraintes en vitesses et on a trouvé que, dans la plage de vitesses qu'on a explorée, on avait une évolution qui était plutôt un peu inférieure à $\mathrm{N}^{2}$. Je ne sais pas si vous avez remarqué que vous aviez une bonne transposition en fonction de $\mathrm{N}^{2}$ sur les fluctuations de contrainte.

Pour résumer mes questions, d'une part avez-vous mesuré ce qui se passait à très bas débit en contrainte et, d'autre part, avez-vous vu comment évoluaient les fluctuations de contrainte en fonction de la vitesse de rotation?

M. MARTIGNAC: On avait trois vitesses de rotation. A 4000 et 6000 , on arrivait à avoir, aux précisions de mesure près, des corrélations en $\mathrm{N}^{2}$ satisfaisantes, à $10 \%$ près environ. Par contre, à 8000 tours, on a obtenu des signaux qui sont plus ou moins moyens. Nous pensons faire une exploitation complémentaire des essais qui peut nous permettre d'avoir confirmation ou infirmation des valeurs que l'on a à l'heure actuelle. Il est aussi nécessaire pour nous d'extrapoler à $15000 \mathrm{t} / \mathrm{min}$.

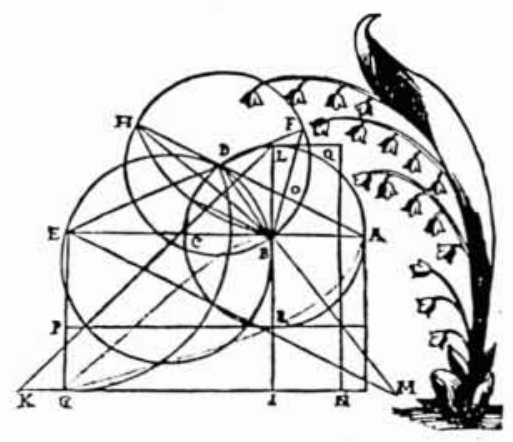

